\title{
Biologia de Automeris illustris (Walker) (Lepidoptera, Saturniidae, Hemileucinae)
}

\author{
Alexandre Specht ${ }^{1}{ }^{2}$; Aline C. Formentini ${ }^{1} \&$ Elio Corseuil ${ }^{3}$ \\ ${ }^{1}$ Laboratório de Biologia, Departamento de Ciências Exatas e da Natureza, Campus Universitário da Região dos Vinhedos, \\ Universidade de Caxias do Sul. Caixa Postal 32, 95700-000 Bento Gonçalves, Rio Grande do Sul, Brasil. E-mail: \\ aspecht@ucs.br; acformen@ucs.br \\ 2 Instituto de Biotecnologia, Universidade de Caxias do Sul. Cidade Universitária, Caixa Postal 1352, 95070-560, Caxias do \\ Sul, Rio Grande do Sul, Brasil. \\ ${ }^{3}$ Laboratório de Entomologia, Faculdade de Biociências, Pontifícia Universidade Católica do Rio Grande do Sul. Caixa postal \\ 1429, 90619-900 Porto Alegre, Rio Grande do Sul, Brasil. E-mail: corseuil@pucrs.br
}

\begin{abstract}
Biology of Automeris illustris (Walker) (Lepidoptera, Saturniidae, Hemileucinae). This work aimed to study the biology of Automeris illustris (Walker, 1855) which is a polyphytophagous, considered secondary pest of eucalypts culture in wich their caterpillars might cause erucism. The biological parameters were obtained in controlled conditions of temperature: $25 \pm 1^{\circ} \mathrm{C}$, UR $70 \pm 10 \%$ and photofase of 14 hours, with daily observations. On each developmental phase, morphological and ethologic aspects, as well as duration and viability, were evaluated. In order to link the host plants to the insect it were added data of collected material on field and referred in the bibliography. Under laboratory conditions it was observed that the life cycle needed of about 121 days whose mean periods of eggs, caterpillars, pre-pupae, pupae and adult phases were 10.60, 80.56, 3.58, 19.17 and 7.83 days, respectively. The caterpillars passed by six instars with a growth average rate of 1.47 . It was observed a high degree of polyphytophagy over 51 host plants belonging in 28 families. The biotic potential was estimated on 8.719.556 individuals in the weak.
\end{abstract}

KEY WORDS. Biological parameters; caterpillars; erucism; life cycle.

RESUMO. Objetivou-se estudar a biologia de Automeris illustris (Walker, 1855), um hemileucíneo polifitófago considerado praga secundária da eucaliptocultura e cujas lagartas podem provocar erucismo. Os parâmetros biológicos foram obtidos em condições controladas de temperatura: $25 \pm 1^{\circ} \mathrm{C}$, UR $70 \pm 10 \%$ e fotofase de 14 horas, com observações diárias. Foram avaliados, em cada fase de desenvolvimento, aspectos morfológicos e etológicos, duração e viabilidade. Para relacionar as plantas hospedeiras foram reunidos dados de material coletado em campo e já referidos em bibliografia. Nas condições de laboratório observou-se que o ciclo de vida necessitou de aproximadamente 121 dias, cujos períodos médios das fases de ovo, lagarta, pré-pupa, pupa e adulta foram de 10,60; 80,56; 3,58; 19,17 e 7,83 dias, respectivamente. As lagartas passaram por seis ínstares e tiveram uma razão média de crescimento de 1,47. Observou-se um alto grau de polifitofagia sendo relacionadas 51 plantas hospedeiras pertencentes a 28 famílias. O potencial biótico foi estimado em 8.719 .556 indivíduos ao ano. PALAVRAS-CHAVE. Ciclo de vida; erucismo; lagartas; parâmetros biológicos.

O gênero Automeris Hübner, [1819] é o mais diverso da subfamília Hemileucinae (Lepidoptera: Saturniidae), representado por 135 espécies que incluem mariposas pequenas até grandes, que normalmente apresentam como peculiaridade uma mancha ocelar conspícua na superfície superior da asa posterior e uma mancha circular quase tão larga na porção inferior da asa anterior. Este gênero é também o mais amplamente distribuído, ocorrendo desde o Canadá até a Argentina (Lemaire 2002).

Entre as espécies mais comuns encontra-se Automeris illustris (Walker, 1855) popularmente conhecida como "olho de pavão alaranjado" tanto no Brasil (Buzzi 1994) como em outros países sul americanos (KÖHLER 1935); apresenta como sinônimos Io pylades Boisduval 1875; Io coffeae Boisduval, 1875; Io phales Boisduval, 1875 e Automeris pelotas Strand, 1920 (Lemaire 2002). Segundo Lemaire (2002) esta mariposa é amplamente distribuída no sudeste do Brasil e adjacências do Uruguai e Argentina com extensão ainda não delimitada ao norte com relação à abrangência dos estados da Bahia até Goiás. É referida em plantios de eucalipto em Açailândia, Maranhão por ZANUNCio et al. (1992) e para outros estados do Norte, Alagoas e Ceará por SiLvA et al. (1968). KÖHLER (1935) refere esta espécie também para o México, Bolívia e Honduras; entretanto, como observado com o 
registro de Guagliumi (1957) para a Venezuela, corrigido por Lemaire (1996) como Automeris liberia (Cramer, 1780), provavelmente este lepidóptero tenha sido confundido com outros similares, ocorrentes naqueles países.

Esta espécie apresenta várias plantas hospedeiras e tem sido referida em obras brasileiras, cujos dados encontram-se reunidos no Quarto Catálogo dos Insetos que Vivem nas Plantas do Brasil (SiLva et al. 1968); têm sido reportada também por estudos sobre lepidópteros nocivos à cultura do eucalipto, sendo considerada uma praga secundária (ZANUNCIO 1993). No Brasil ainda é referida em estudos que tratam da entomofauna restrita a regiões (MARINONi et al. 1997). Subsídios também são encontrados em obras relativas a entomofauna americana (DrAUDT 1929, LEMAIRE 2002), neotropical (Lemaire 1996), ou de países vizinhos como Argentina (KöHLer 1935) e Uruguai (Biezanko et al. 1957, 1974).

Com relação à sua ocorrência MARINONI et al. (1997) referem esta espécie em oito localidades representativas do estado do Paraná em observações feitas no período de outubro de 1986 a setembro de 1987; da mesma forma, é referida como uma das espécies-praga secundárias mais coletadas em diferentes regiões do Espirito Santo, Minas Gerais e São Paulo (Zanuncio et al. 1992, 1994, 1998a, b, SAntos et al. 1996a) e, no Rio Grande do Sul representada na maioria das coleções entomológicas (CoRseuil et al. 2002). É facilmente criada em laboratório (GARDINER 1982) e já foi utilizada em diversos experimentos sobre fisiologia de insetos na Europa (Tabouret-Keller 1958, Collett 1965, Blest \& Collett 1965).

Suas lagartas, à semelhança das pertencentes aos demais representantes do gênero, são urticantes (Lemaire 2002, HadDAD \& Cardoso 2003, Moraes 2003) e por serem comuns são freqüentemente trazidas pelos alunos do Campus Universitário da Região dos Vinhedos, Universidade de Caxias do Sul, recolhidas por vítimas de acidentes.

Considerando-se a importância e a inexistência de estudos detalhados, este trabalho teve por objetivo determinar os principais aspectos biológicos desta espécie com observações em laboratório e em campo.

\section{MATERIAL E MÉTODOS}

A criação laboratorial foi feita em sala climatizada sob temperatura de $25 \pm 1^{\circ} \mathrm{C}$, umidade relativa de $70 \pm 10 \%$ e fotofase de 14 horas, com observações diárias. Iniciada a partir de duas posturas provenientes de uma fêmea coletada pela segunda autora, em Bento Gonçalves, Rio Grande do Sul, em 10 de janeiro de 2003.

Os aspectos morfológicos referentes às fases de desenvolvimento foram avaliados efetuando-se mensurações através de paquímetro digital e microscópio estereoscópico provido de ocular micrométrica com precisão de centésimo de milímetro e balança com precisão de centésimo de grama.

Fase de ovo. As posturas foram individualizadas em placas de petri cujo fundo foi forrado com papel filtro umedecido com água destilada, onde permaneceram até a eclosão das la- gartas. Além de observações morfológicas foram efetuadas mensurações do maior comprimento e largura.

Fase de lagarta. Inicialmente 150 lagartas foram mantidas isoladamente em recipientes de PVC com capacidade de $300 \mathrm{~mL}$, para observações individuais; seus resultados deixaram de ser considerados porque, em função do comportamento gregário, nenhum exemplar atingiu a fase adulta. Em função disso, as demais lagartas foram criadas em 10 grupos provenientes de posturas que variaram de 56 a 128 ovos, mantidas em gaiolas de PVC, cilíndricas de $19 \mathrm{~cm}$ de diâmetro por 23 de altura. Foram alimentadas com folhas de corticeira-do-banhado, Erythrina crista-galli Linnaeus - Fabaceae, disponibilizada em ramos, renovados diariamente, cujas extremidades foram mantidas em água destilada para manter a turgescência. Para a diferenciação dos instares, como referido em PARRA \& HADDAD (1989), organizou-se uma distribuição de freqüência das medidas das cápsulas cefálicas recolhidas durante a criação. Ao final das medições, avaliou-se a razão de crescimento comparando a sua adequação ao modelo linear de Dyar (1890). Ainda foram efetuadas observações etológicas.

Período de pré-pupa. Considerou-se terem atingido o período de pré-pupa quando as lagartas pararam de se alimentar e se dispersaram procurando um local para a construção do casulo. Neste momento foram individualizadas em recipientes de vidro com capacidade de $500 \mathrm{ml}$, com papel filtro umedecido com água destilada no fundo, e tapados com tecido voile branco, na extremidade superior. Realizou-se uma pequena incisão nos casulos para constatar a data da formação das pupas e registrar a duração do período.

Fase de pupa. No segundo dia após a formação, quando a cutícula já estava mais endurecida os casulos foram abertos e as pupas foram removidas para a verificação do sexo, seguindo-se esquemas de ButT \& CANTU (1962); além da duração desta fase foram avaliados o peso, a maior largura e o comprimento.

Fase adulta. Após a emergência foram formados 20 casais com mariposas de mesma idade, mantidos em gaiolas cilíndricas de PVC, com diâmetro e altura de $20 \mathrm{~cm}$, revestidas com filme plástico na base e voile branco na porção superior. Estas gaiolas foram forradas, no fundo e nas laterais, com papel filtro, diariamente umedecido com água destilada e também receberam um graveto da planta hospedeira para a oviposição. Foram avaliados os parâmetros envergadura, longevidade, períodos de pré, pós e oviposição.

A partir da reunião dos parâmetros biológicos calculou-se o potencial biótico seguido-se a fórmula constante em SiLveira Neto et al. (1976) considerando-se nula a resistência do ambiente.

Os dados foram analisados através de estatística descritiva com o cálculo das médias e erros-padrão. Quando necessário as médias foram comparadas pelo teste $t$, presumindo variâncias diferentes ao nível de significância de 95\%.

Exemplares testemunhos da pesquisa foram incorporados à coleção do Laboratório de Biologia, Campus Universitário da Região dos Vinhedos, Universidade de Caxias do Sul (CARVI-UCS). 
Além da criação laboratorial para obtenção dos principais parâmetros biológicos realizou-se levantamento das plantas hospedeiras citadas em bibliografia e foram reunidas informações sobre plantas hospedeiras, principalmente da região nordeste do Rio Grande do Sul, a partir de lagartas trazidas por estudantes e comunidade geral ao Laboratório de Biologia do CARVI-UCS nos últimos três anos, cujos exemplares foram mantidos em criação, sempre que possível na própria planta hospedeira. A nomenclatura das plantas foi atualizada utilizando-se, principalmente, BACKES \& NARDINo (2001) relacionando-se os nomes vulgares, científicos, família e referências (Tab. I).

Com vistas a obter dados sobre a época de ocorrência dos adultos foram examinados materiais depositados nas coleções sediadas no Rio Grande do Sul: Centro de Pesquisa Agropecuária de Clima Temperado (CAMB), Museu de Ciências da Universidade Católica de Pelotas (MUCP) e Museu Entomológico Ceslau Biezanko (MECB) em Pelotas; Museu Anchieta de Porto Alegre (MAPA), Museu de Ciências e Tecnologia da PUCRS (MCTP), Museu de Ciências Naturais da Fundação Zoobotânica do Rio Grande do Sul (MCNZ), Museu Ramiro Gomes Costa da Fundação Estadual de Pesquisa Agropecuária (MRGC) em Porto Alegre e Coleção do Laboratório de Biologia CARVI-UCS (CUCS), em Bento Gonçalves.

Material examinado. Brasil, Rio Grande do Sul: Iraí, 24.III.1998, J.A. Teston leg., 1 macho (MCTP-11058); 24.III.1998, J.A. Teston leg., 1 macho (MCTP-11059); 24.III.1998, J.A. Teston leg., 1 macho (MCTP-11060); 24.III.1998, J.A. Teston leg., 1 macho (MCTP-11061); 26.III.1998, J.A. Teston leg., 1 fêmea (MCTP11057); Derrubadas, 24.I.2001, sem coletor, 7 machos (MCTP10395); Barracão, 21.II.2001, sem coletor, 3 machos (MCTP10396); Cerro Largo, II.1943, sem coletor, 1 macho (MAPA); Cerro Largo (antes Serro Azul), X.1938, sem coletor, 1 fêmea (MAPA); I.1933 sem coletor, 1 macho (MAPA); Guarani das Missões (antes Guarani), 10.I.1933, C.M. Biezanko leg., 1 macho (MECB); Anta Gorda 17.I.2004, 1 fêmea, E. Fronza leg. (CUCS); Bento Gonçalves 28.IV.2003, 1 fêmea, A. Specht leg. (CUCS); 28.V.2003, 1 fêmea, M. Gedoz leg. (CUCS); 12.VI.2003, 1 fêmea, V. Sganzerla leg. (CUCS); 18.II.2004, 1 fêmea, A.C. Formentini leg. (CUCS); 20.III.2004, 1 fêmea, A.C. Formentini leg. (CUCS); 16.IV.2004, 1 macho, A.C. Formentini leg. (CUCS); 17.IV.2004, 1 fêmea, A.C. Formentini leg. (CUCS); 22.IV.2004, 1 fêmea, A.C. Formentini leg. (CUCS); 22.IV.2004, 1 macho, A.C. Formentini leg. (CUCS); 23.IV.2004, 1 macho, A.C. Formentini leg. (CUCS); 25.IV.2004, 1 macho, A.C. Formentini leg. (CUCS); 28.V.2004, 1 fêmea, V. Comparin (CUCS); Caxias do Sul, 03.IV.1924, sem coletor, 1 macho (MCNZ-79203); 12.X.1932, sem coletor, 1 fêmea (MCNZ79205); 29.III.2004, 1 macho, A. Gazola leg. (CUCS); Garibaldi 02.V.2003, 1 fêmea, M. Ecker leg. (CUCS); 01.I.2004, 1 macho, M. Gedoz leg. (CUCS); 03.I.2004, 1 fêmea, M. Gedoz leg. (CUCS); 03.I.2004, 1 macho, M. Gedoz (CUCS); 04.I.2004, 1 macho, M. Gedoz leg. (CUCS); 07.I.2004, 1 fêmea, M. Gedoz leg. (CUCS); 29.IV.2004, 1 fêmea, M. Gedoz leg. (CUCS); 28.V.2004, 1 macho, M. Gedoz leg. (CUCS); São Francisco de Paula, 07.II.1997, sem coletor, 1 macho (MCTP-11065); Salvador do Sul (antes São Salvador), XII.1954, sem coletor, 1 macho (MAPA); 12.IX.1995, A. Specht leg., 1 macho (MCTP-11053); 26.XII.1996, A. Specht leg., 1 macho (MCTP-8034); Nova Petrópolis, 11.I.1961, sem coletor, 1 fêmea (MCNZ-79206); Alegrete, 23.XI.2000, R.A. Di Mare leg., 1 macho (MCTP-11066); 24.XI.2000, R.A. Di Mare leg., 1 macho (MCTP-11067); Pareci Novo, 01.III.1926, sem coletor, 1 fêmea (MAPA); 27.VI.1932, sem coletor, 1 macho (MAPA); 18.IX.1932, sem coletor, 1 fêmea (MAPA); 20.IX.1932, sem coletor, 1 fêmea (MAPA); 24.VI.1933, sem coletor, 1 fêmea (MAPA); 19.VI.1938, sem coletor, 1 fêmea (MAPA); 23.V.1939, sem coletor, 1 macho (MAPA); III.1944, sem coletor, 1 fêmea (MAPA); São Leopoldo, 01.X.1932, sem coletor, 1 fêmea (MAPA); XI.1943, sem coletor, 1 macho (MAPA); X.1945, sem coletor, 1 macho (MAPA); Charqueadas, II.1940, sem coletor, 1 macho (MAPA); São Jerônimo, 13-19.VIII.1982, C.J. Becker leg., 1 macho (MCNZ37904); 14.IX.1982, H.A. Gastal leg., 1 macho (MCNZ-38119); 15.IX.1982, H.A. Gastal leg., 1 macho (MCNZ-38263); 12.X.1982, C.J. Becker leg., 1 macho (MCNZ-38267); 13.X.1982, H.A. Gastal leg., 1 macho (MCNZ-38387); 19.X.1982, C.J. Becker leg., 1 macho (MCNZ-38268); Cachoeira do Sul, 27.III.1998, sem coletor, 1 macho (MCTP-11055); 27.III.1998, sem coletor, 1 macho (MCTP-11056); Porto Alegre, 26.VII.1925, sem coletor, 1 macho (MAPA); 08.VIII.1927, sem coletor, 1 macho (MAPA); 03.IV.1938, sem coletor, 1 macho (MAPA); 15.IV.1938, sem coletor, 1 macho (MAPA); 01.IX.1939, sem coletor, 1 fêmea (MCNZ-79204); 28.XI.1940, sem coletor, 1 fêmea (MAPA); II. 1941, C.P. Costa leg., 1 macho (MRGC-3429); XI.1946, sem coletor, 1 fêmea (MAPA); 11.III.1947, sem coletor, 1 fêmea (MAPA); 26.III.1951, sem coletor, 1 fêmea (MAPA); 26.III.1957, sem coletor, 1 fêmea (MAPA); 11.II.1959, E. Corseuil leg., 1 macho (MRGC-3433); 12.II.1959, E. Corseuil leg., 1 fêmea (MRGC-3434); 10.X.1966, sem coletor, 1 macho (MAPA); 04.IX.1967, sem coletor, 1 fêmea (MAPA); 10.IX.1968, sem coletor, 1 fêmea (MAPA); 01.III.1969, sem coletor, 1 macho (MAPA); 19.X.1971, sem coletor, 1 macho (MAPA); 07.VII.1977, sem coletor, 1 fêmea (MAPA); 14.II.1995, L. Lorini leg., 1 macho (MCTP-11054); Viamão (bairro Agronomia), 09.II.1966, sem coletor, 1 macho (MAPA); Caçapava do Sul, 30.II.2003, 1 macho, M. Rossato leg. (CUCS); Pelotas, 15.IV.1938, C.M. Biezanko leg., 1 fêmea (MECB); 05.X.1938, C.M. Biezanko leg., 1 macho (MRGC-3426); 21.XII.1938, C.M. Biezanko leg., 1 macho (MECB); 26.IV.1939, irmãs Figueiredo leg., 1 macho (MUCP); 20.X.1939, C.M. Biezanko leg., 1 fêmea (MECB); 14.XI.1939, irmãs Figueiredo leg., 1 fêmea (MUCP); 25.XI.1941, irmãs Figueiredo leg., 1 fêmea (MUCP); 02.X.1946, C.M. Biezanko leg., 1 macho (MECB); 11.II.1947, irmãs Figueiredo leg., 1 macho (CAMB); 15.II.1947, irmãs Figueiredo leg., 1 macho (CAMB); 24.II.1948, C.M. Biezanko leg., 1 macho (MECB); 07.IV.1948, Irmãs Figueiredo leg., 1 fêmea (CAMB); 07.XII.1949, sem coletor, 1 macho (CAMB); 23.VIII.1961, sem coletor, 1 macho (CAMB); 30.IV.1982, C. Jusreti leg., 1 macho (MECB); 26.XI.1982, M.A. Pagman leg., 1 macho (MECB); Rio Grande, I.1933, irmãs Figueiredo leg., 1 macho (MUCP).

Revista Brasileira de Zoologia 23 (2): 537-546, junho 2006 
Tabela I. Plantas hospedeiras de Automeris illustris segundo vários autores e dados obtidos durante o presente estudo.

\begin{tabular}{|c|c|c|c|}
\hline Nome vulgar & Nome científico & Família & Referência \\
\hline Abacateiro & Persea americana Mill & Lauraceae & 2 \\
\hline Acácia & Acacia spp. & Mimosaceae & 2 \\
\hline Acácia-aroma & Acacia dealbata Link & Mimosaceae & 3,4 \\
\hline Acácia-mole & Acacia mollissima (Willdenow) Lindley & Mimosaceae & 3 \\
\hline Algodoeiro & Gossypium barbadense Linnaeus & Malvaceae & 2 \\
\hline Araçazeiro & Psidium araca Raddi & Myrtaceae & 2 \\
\hline Araçazeiro-amarelo & Psidium cattleyanum Sabine & Myrtaceae & 4 \\
\hline Bananeira & Musa paradisiaca Linnaeus & Musaceae & 6 \\
\hline Cafeeiro & Coffea arabica Linnaeus & Rubiaceae & 1,2 \\
\hline Candiuba & Trema micrantha (Linnaeus) Blume & Ulmaceae & 2 \\
\hline Caquizeiro & Diospyros kaki Linnaeus & Ebenaceae & 6 \\
\hline Cinamomo & Melia azedarach Linnaeus & Meliaceae & 6 \\
\hline Citros & Citrus spp. & Rutaceae & 2 \\
\hline Cocão & Erytroxylum cuneifolium (Martius) O. E. Schulz & Erythroxylaceae & 6 \\
\hline Corticeira-do-banhado & Erythrina crista-galli Linnaeus & Fabaceae & 6 \\
\hline Corticeira-da-Serra & Erythrina falcata Bentham & Fabaceae & 6 \\
\hline Crataegus & Crataegus sp. & Rosaceae & 5 \\
\hline Erva-mate & Ilex paraguariensis Saint Hilaire & Aquifoliaceae & 4 \\
\hline Ervilha & Psidum sativum Linnaeus & Fabaceae & 2 \\
\hline Escumilha & Lagestroemia speciosa (Linnaeus) Banaba & Lythraceae & 2 \\
\hline Espinilho & Acacia caven (Molina) Molina & Mimosaceae & 2 \\
\hline Esponja & Acacia farnesiana (Linnaeus) Willdenow & Mimosaceae & 2 \\
\hline Extremosa & Lagerstroemia indica Linnaeus & Lythraceae & 2 \\
\hline Eucalipto & Eucalyptus sp. & Myrtaceae & 2 \\
\hline Fagus & Fagus sp. & Fagaceae & 5 \\
\hline Fícus & Ficus retusa Linnaeus var. nitida (Th.) Miqquel & Moraceae & 2 \\
\hline Fícus & Ficus benjamina Linnaeus & Moraceae & 5 \\
\hline Glicínia & Wistaria sinensis Sweet & Fabaceae & 3 \\
\hline Goiabeira & Psidium guajava Linnaeus & Myrtaceae & 2 \\
\hline Goiabeira-da-serra & Acca sellowiana (Berg.) Burret & Myrtaceae & 6 \\
\hline Guandeiro & Cajanus indicus Spreng & Fabaceae & 2 \\
\hline Hibisco & Hibiscus rosa-sinensis Linnaeus & Malvaceae & 6 \\
\hline Ingá-banana & Inga uruguensis Hooker et Arnott & Mimosaceae & 3 \\
\hline Ingá-macaco & Inga sessilis Martius & Mimosaceae & 4 \\
\hline Ingazeiro & Inga sp. & Mimosaceae & 2 \\
\hline Jacarandá & Jacaranda mimosifolia D. Don & Bignoniaceae & 6 \\
\hline Jasmineiro-azul & Plumbago capensis Thumberg & Plumbaginaceae & 2 \\
\hline Laranjeira-doce & Citrus sinensis (Linnaeus) Osbeck & Rutaceae & 3 \\
\hline Ligustro* & Ligustrum sp. & Oleaceae & 5 \\
\hline Lilás & Syringa vulgaris Linnaeus & Oleaceae & 5 \\
\hline Madressilva & Lonicera japonica Thumberg & Caprifoliaceae & 2 \\
\hline Mangueira & Mangifera indica Linnaeus & Anacardiaceae & 2 \\
\hline Magnolia & Magnolia sp. & Magnoliaceae & 1 \\
\hline Mirindiba & Lafoensia glyptocarpa Koohne & Lythraceae & 2 \\
\hline Pata-de-vaca & Bauhinia forficata Link & Caesalpiniaceae & 6 \\
\hline Pereira & Pyrus commnunis Linnaeus & Rosaceae & 6 \\
\hline Pingo-de-ouro & Duranta repens Linnaeus var. aurea Hort & Verbenaceae & 6 \\
\hline Plátano-oriental & Platanus orientalis Linnaeus & Platanaceae & $2,3,5$ \\
\hline Roseira & Rosa spp. & Rosaceae & 2 \\
\hline \multirow[t]{2}{*}{ Salseiro-chorão } & Salix babylonica Linnaeus & Salicaceae & 2,3 \\
\hline & & Melastomataceae & 1 \\
\hline
\end{tabular}

1) Draudt (1929); 2) Silva et al. (1968); 3) BieZanko et al. (1974); 4) BieZanko (1986); 5) Stone (1991), 6) observações do presente estudo.

Revista Brasileira de Zoologia 23 (2): 537-546, junho 2006 


\section{RESULTADOS E DISCUSSÃO}

Os ovos (Fig. 1), de formato subcilíndrico, levemente achatados longitudinalmente, ficam presos ao substrato, normalmente um ramo, pelo pólo mais estreito, oposto ao que contém a micrópila, que é mais plano. A coloração é branca e a micrópila é verde-escura, principalmente no centro; tornando-se negra próximo à eclosão das lagartas porém com um anel esverdeado ao seu redor. O maior comprimento do eixo micropilar é 1,766 \pm $0,021 \mathrm{~mm}$ e a maior largura 1,642 $\pm 0,020 \mathrm{~mm}(\mathrm{n}=10, \mathrm{p}<0,05)$. Apesar dos ovos serem dispostos em grupos, um ao lado do outro, tocando-se em um ou outro ponto, ficavam aderidos apenas ao substrato. De maneira geral os ovos desta espécie se parecem muito com os descrito para várias espécies do gênero (Lemaire 2002) e, especialmente da descrição feita por BourQuin (1948) para Automeris naranja Schaus, 1898, que são pouco menores.

Das primeiras posturas obteve-se viabilidade de 100\%, entretanto, na geração seguinte a viabilidade foi de $88,42 \% \pm$ 11,34 ( $\mathrm{n}=12$ posturas, 567 ovos). Verificou-se que, em laboratório, a fertilidade foi menor do que a observada com os ovos provenientes do adulto coletado na natureza. Fatores como a presença de apenas um casal por gaiola podem ter interferido negativamente pois na criação de hemileucíneos como Hylesia nanus (Walker, 1855) foi constatado que a presença de apenas um casal de adultos por gaiola proporcionou fertilidade muito baixa, quando comparado com a presença de dois ou três casais (SANTos et al. 1996b). Entretanto, este valor aproxima-se dos apresentados por LoRINi et al. (2004) para outro hemileucíneo, Lonomia obliqua Walker, 1855 em condições de laboratório.

O tempo de incubação (Tab. II) foi bastante inferior ao descrito para $A$. naranja (27 dias) à temperatura de $22^{\circ} \mathrm{C}$ (BourquiN 1948) e de L. obliqua, onde já foi observado em torno de 17 (LORINI 1999) a 38 dias em diferentes temperaturas (LoRINI et al. 2004).

Tabela II. Duração média em dias e erros padrões das fases de desenvolvimento de Automeris illustris criada a $25 \pm 1{ }^{\circ} \mathrm{C}$, UR $70 \pm 10 \%$ e $14 \mathrm{~h}$ fotofase.

\begin{tabular}{llr}
\multicolumn{1}{c}{ Fases } & $\mathrm{N}$ & \multicolumn{1}{c}{ Média } \\
\hline Ovo (posturas) & 10 & $10,60 \pm 0,41$ \\
Lagarta & 27 & $80,56 \pm 1,15$ \\
Pré-pupa & 19 & $3,58 \pm 0,12$ \\
Pupa & 18 & $19,17 \pm 0,32$ \\
Adulta (casais) & 20 & $7,83 \pm 0,19$ \\
Pré-oviposição & 16 & $6,31 \pm 0,24$ \\
Oviposição & 16 & $1,25 \pm 0,11$ \\
Pós-oviposição & 16 & $0,44 \pm 0,13$ \\
\hline
\end{tabular}

Ao eclodirem, as lagartas alimentaram-se do córion dos ovos que é referido como o primeiro alimento de todos os hemileucíneos após a eclosão (Lemaire 2002) posteriormente procuraram em fila um substrato, normalmente a borda inferi- or de uma folha, onde permaneceram agrupadas por um dia sem se alimentarem. Desde a eclosão até o terceiro instar observou-se gregarismo acentuado em todas as agregações mantidas em laboratório, o que é bastante comum entre os representantes de Hemileucinae (Lemaire 2002).

Desde o primeiro instar as lagartas apresentam a coloração de fundo e cabeça verde-claro, até o final do desenvolvimento (Lemaire 2002). Entretanto, no primeiro instar (Fig. 2) podem ser vistas faixas longitudinais conspícuas e máculas que se tornarão escuras, presentes entre os escolos supra espiraculares e dorsais. Os escolos têm a mesma coloração do corpo exceto os do pronoto pouco mais escuros.

As lagartas passaram por seis ínstares (Tab. III), observando-se um grande aumento na variação entre as larguras das cápsulas com o desenvolvimento, que deve ser atribuído, principalmente, à diferenciação sexual, com fêmeas progressivamente maiores que os machos, especialmente após o quarto instar. Apesar de não fornecer medidas para a fase de lagarta, Lemaire (2002) refere a ocorrência de seis ínstares para esta espécie.

Tabela III. Médias da largura da cápsula cefálica em milímetros com respectivo erro padrão e razão de crescimento, de lagartas de Automeris illustris alimentadas com folhas de corticeira-dobanhado (Erythrina crista-galli), em laboratório.

\begin{tabular}{cccc}
\hline Instar & $\mathrm{N}$ & $\begin{array}{c}\text { Largura da cápsula } \\
\text { cefálica }(\mathrm{mm})(\mathrm{X} \pm \mathrm{EP})\end{array}$ & $\begin{array}{c}\text { Razão de } \\
\text { Crescimento }\end{array}$ \\
\hline I & 132 & $0,90 \pm 0,03$ & - \\
II & 122 & $1,32 \pm 0,05$ & 1,47 \\
III & 110 & $1,95 \pm 0,07$ & 1,48 \\
IV & 92 & $2,87 \pm 0,11$ & 1,47 \\
V & 87 & $4,23 \pm 0,36$ & 1,47 \\
VI & 23 & $6,19 \pm 0,45$ & 1,46 \\
\hline
\end{tabular}

Observou-se uma razão média de crescimento de 1,47 (Tab. III) para as cápsulas cefálicas, entre os ínstares, sendo coerente com o previsto pela regra de DyAR (1890).

De 418 lagartas acompanhadas, 410 atingiram o estágio de pré pupa, obtendo-se uma viabilidade de 98,09\%. A duração de pupa (Tab. II) foi vinte dias, sendo mais longa que a observada para $A$. naranja criada em temperatura média de $26^{\circ} \mathrm{C}$ (Bourquin 1948) e um pouco menor que os 90 dias estimados por LoRINI (1999) para Lonomia obliqua Walker, 1855, criada em condições naturais.

Ao final do desenvolvimento (Figs 3-4), conforme descrito em Gardiner (1982) e Lemaire (2002) podem ser vistos os pares de escolos supra-espiraculares e dorsais muito desenvolvidos em cada segmento e os subespiraculares pouco conspícuos por serem muito menores. O espiráculos são alaranjados e abaixo deles observa-se uma linha negra tênue, interrompida a cada segmento, sobre outra branca larga e nunca interrompida. Abaixo desta linha branca, entre os segmentos abdominais, 


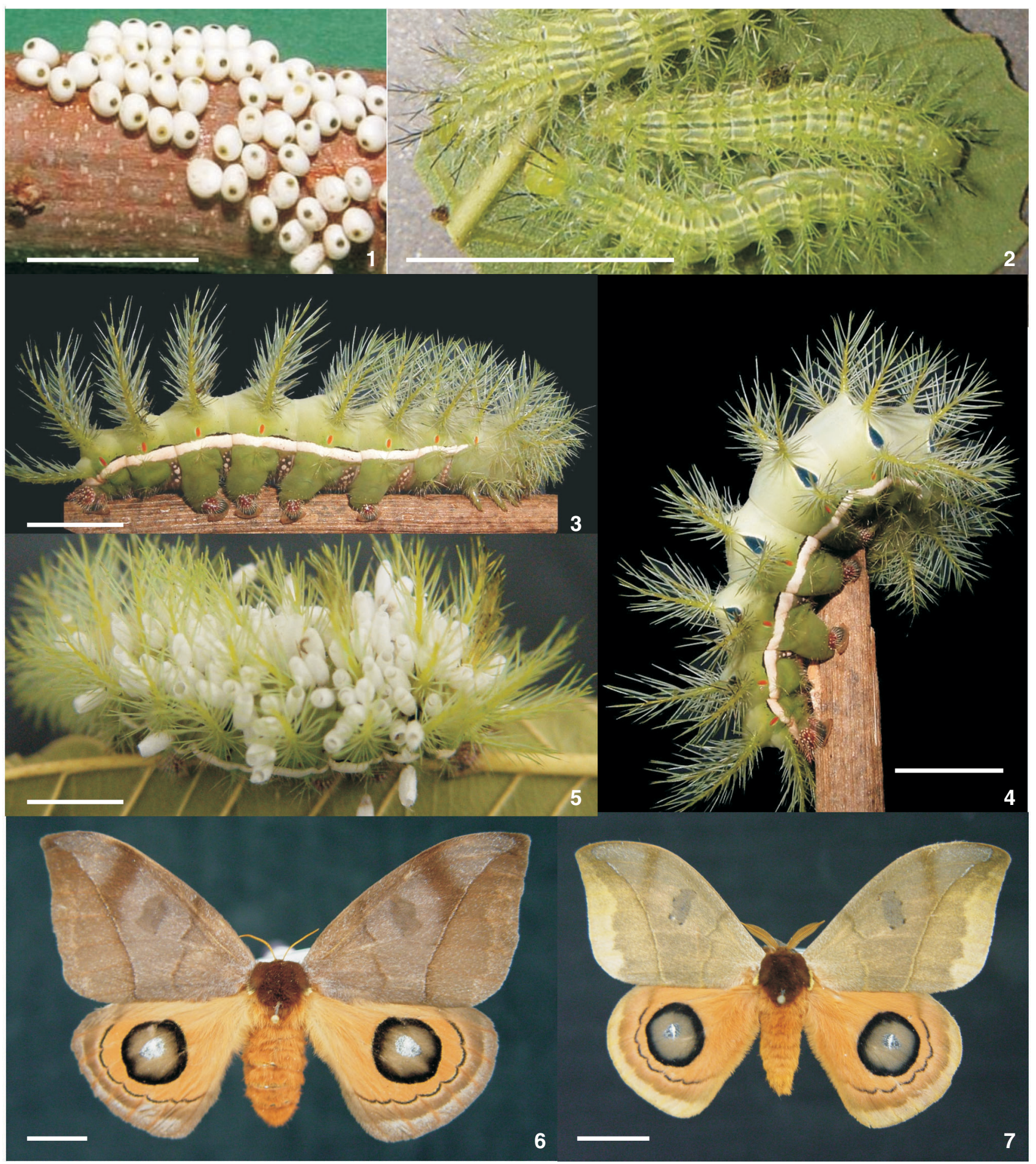

Figuras 1-7. Automeris illustris: (1) ovos; (2) lagartas de primeiro ínstar; (3) lagarta de último instar em repouso; (4) lagarta de último ínstar em posição de defesa; (5) lagarta parasitada; (6) fêmea adulta; (7) macho adulto. Barra $=1 \mathrm{~cm}$.

Revista Brasileira de Zoologia 23 (2): 537-546, junho 2006 
especialmente entre os pseudópodos, encontra-se uma área escura, as vezes de coloração roxa, com pintas brancas. As lagartas de último instar, especialmente as que originarão fêmeas são grandes, podendo atingir mais de $7 \mathrm{~cm}$ de comprimento.

Quando em repouso (Fig. 3) as lagartas mantêm os escolos maiores eretos sobre a região dorsal do corpo como descrito em Lemaire (2002). Ao serem perturbadas contraem o corpo, muitas vezes arqueando-se ventralmente, direcionando os escolos supraespiraculares lateralmente, destacando-se as manchas escuras muito conspícuas entre estes e os escolos dorsais em cada segmento (Fig. 4).

Ao realizar a troca de alimento observou-se que ao serem perturbadas, as lagartas contraiam o corpo e eriçavam os escolos e emitiam um ruído ou estridulação semelhante a um estalo. Após alguns exemplares da gaiola de criação emitirem os estalos todas as lagartas do mesmo recipiente tomavam posição defensiva, como um efeito cascata; muitas delas se desprendiam da planta hospedeira, contorcendo-se agressivamente, provavelmente como uma reação comportamental que visa intimidar o agressor. Com relação à emissão de ruídos e resposta comportamental a estímulos sonoros YACK et al. (2001), exemplificando com diversos táxons, demonstraram evidências de que é uma forma de comunicação muito difundida entre os lepidópteros, considerada como a principal forma de comunicação larval do grupo.

Apesar de não ser observado canibalismo entre as lagartas, por diversas vezes foram vistas comendo exúvias, concordando com o comportamento descrito por Lemaire (2002), para os representantes de Hemileucinae em geral.

Durante o dia, no campo e por diversas vezes, observou-se que as lagartas permanecem presas à margem ventral das folhas, em grupos ou em duplas quando pequenas, ou individualmente quando maiores, desta forma passam desapercebidas pelos predadores. Provavelmente seja a forma mais utilizada por este inseto para camuflar-se, principalmente em função da sua coloração que se confunde com o verde das folhas. Outros hemileucíneos que apresentam coloração mais escura ou que não são verdes, como os representantes de Lonomia Walker, 1855 utilizam-se também da coloração para confundirem-se com o meio, especialmente em troncos com liquens ou musgos onde permanecem a maior parte do dia em agregações (LoRINI 1999).

A reunião dos dados referentes às plantas hospedeiras permitiu relacionar 51 plantas hóspedes pertencentes a 28 famílias vegetais (Tab. I), destacando-se os representantes de Mimosaceae, Myrtaceae e Fabaceae. O registro de grande variedade de hospedeiros indica facilidade de adaptação a diferentes grupos vegetais relacionando-se esta espécie como polifitófaga. Segundo Lemaire (2002), apesar de existirem espécies monófagas, a polifitofagia é comum entre os representantes deste gênero, destacando que para espécies mais conhecidas como Automeris io (Fabricius, 1775) existe a indicação de 120 espécies incluídas em 34 famílias (STONe 1991); observando-se a ocorrência de variações em preferências alimentares locais (Lemarre 2002).

Apesar de polifitófaga observou-se que as lagartas dificil- mente mudam de planta hospedeira durante o seu desenvolvimento, o caso mais expressivo foi de um exemplar coletado no município de Anta Gorda, RS, 25.V.2003, pelo Biólogo Edegar Fronza em folhas de bananeira, Musa paradisiaca Linnaeus Musaceae. A lagarta de quinto instar foi colocada em um recipiente com diversas plantas que habitualmente são utilizadas como hospedeiras, sem sucesso, o exemplar só voltou a comer quando oferecidas folhas de bananeira, cinco dias depois. JANZEN (2003), descrevendo padrões alimentares de lagartas da Costa Rica, divide as espécies generalistas em dois grupos: um que se alimenta de 5 a 10 espécies de plantas aparentadas até completamente não relacionadas em um ecossistema natural, mas cada lagarta individualmente alimenta-se apenas da planta hospedeira utilizada pela sua mãe para ovipositar; no outro caso, uma lagarta alimenta-se de 5 a 10 plantas hospedeiras diferentes, deslocando-se de planta para outra, fugindo do ataque de predadores e não retornando para a planta original, neste caso a migração normalmente ocorre no penúltimo e último ínstares. Em cinco casos, em função de não haver disponibilidade da planta hospedeira em que a lagarta havia sido coletada não se obteve sucesso na criação; em três casos, todos envolvendo mirtáceas, conseguiu-se obter adultos modificando a planta hóspede original por goiabeira Psidium guajava Linnaeus Myrtaceae. Em função destas observações A. illustris pode ser enquadrada na primeira categoria de lagartas polifitófagas.

Na fase de pré-pupa, caracterizada pela parada da atividade de alimentação e a procura de um lugar para a confecção do casulo,observou-se que as lagartas tornaram-se mais escuras, e reduziram de tamanho. Utilizaram pelo menos metade do período desta fase (Tab. II) para confeccionar envoltórios de parede única, parcialmente resistentes e impermeáveis, não bem caracterizados como casulos, utilizando fios grosseiros de seda de cor âmbar, unindo folhas ou outros restos vegetais. Este tipo de construção concorda com a descrição de MABILdE (1896) e dos casulos de Automeris por Lemaire (2002)

Das 410 pré-pupas obtiveram-se 383 pupas sem deformação, observando-se uma viabilidade de 93,41\%; tais resultados sugerem que esta seja a fase mais crítica do desenvolvimento pós-embrionário da espécie.

Logo após a ecdise, as pupas apresentaram-se moles com coloração verde clara, foram tornando-se avermelhadas e endurecidas, em menos de quatro horas, a cutícula já estava completamente endurecida e a coloração castanho escura. Nesta fase as fêmeas $(n=35)$ foram significativamente maiores que os machos $(\mathrm{n}=35$ ) com massa de 4,846 $\pm 0,093 \mathrm{~g}$ e 4,728 $\pm 0,881 \mathrm{~g}$; comprimento de $37,672 \pm 0,269 \mathrm{~mm}$ e $35,097 \pm 0,321 \mathrm{~mm}$; largura de $14,963 \pm 0,147 \mathrm{~mm}$ e $14,416 \pm 0,610 \mathrm{~mm}$, respectivamente. Tais diferenças eram esperadas em função do grande dimorfismo sexual característico do grupo, também relatado para esta espécie (Lemaire 2002).

Não houve diferença significativa para a duração desta fase entre machos e fêmeas (Tab. II), entretanto, nas condições deste estudo a duração foi menor que a constante em MABILDE (1896)

Revista Brasileira de Zoologia 23 (2): 537-546, junho 2006 
que menciona 1, 2 ou 6 meses. A ocorrência de um longo período pupal referida pelo último autor pode estar relacionada com a capacidade de passar por diapausa pupal, já citada para alguns representantes de Hemileucinae (Lemaire 2002). Bourquin (1944), na Argentina, relata que Automeris coresus (Boisduval, 1859) uma espécie muito próxima de $A$. illustris, passa o inverno na fase de pupa. Com relação a isto, Lemarre (2002) ainda cita que, após a fase de ovo, esta é a segunda forma mais comum destes lepidópteros passarem o inverno no hemisfério norte. Acrescenta ainda que, em determinados locais, populações bivoltinas de A. io apresentam uma geração sem e outra com diapausa que atravessa o inverno, sem fazer alusão ao estágio de pupa.

De 383 pupas emergiram 378 adultos, obtendo-se uma viabilidade de 98,69\%. Destes adultos 191 fêmeas e 187 machos com uma razão sexual de 0,505 lagartas que não difere da proporção que prevê igualdade na proporção entre sexos.

As fêmeas adultas (Fig. 6) foram significativamente maiores com envergadura média de 116,273 $\pm 1,373 \mathrm{~mm}(\mathrm{n}=15)$ enquanto os machos (Fig. 7) apresentaram 99,313 \pm 1,675 mm ( $\mathrm{n}=15)$. O tamanho dos exemplares criados em laboratório foram semelhantes ao descrito por Lemaire (2002) que indica envergadura de 91-122 mm para fêmeas e 89-101 para machos.

Não houve diferenças significativas entre longevidade dos adultos (Tab. II) que é muito curta e, presumivelmente encontra-se relacionada com o fato dos hemileucíneos não se alimentarem durante a fase adulta (Lemaire 2002). O tempo de pré-oviposição relativamente mais extenso que o de oviposição e pós-oviposição, provavelmente está relacionado com a maturação sexual, atividades de atração e localização do sexo oposto, cópula e localização da planta hospedeira para que finalmente seja realizada a oviposição, finalizando o ciclo.

O ciclo de vida completo foi de 121,74 dias o que corresponde a 2,998 gerações anuais. Estes resultados sugerem que dependendo da latitude, como observado para $A$. io que pode apresentar de uma geração ao norte até três ou quatro na Flórida e sudeste do Texas (Lemaire 2002), esta espécie pode apresentar número variável de gerações.

Número médio de 514,75 ovos foi bastante superior ao referido para outros hemileucíneos como H. nanus, em torno de 300 (SANTos et al.1996b) e L. obliqua, em torno de 135 (LoRINI et al. 2004).

Considerando-se a sobrevivência da fase de ovo até adulto a viabilidade foi de $79,87 \%$. Desta forma cada fêmea é capaz de gerar em média 411,65 descendentes. Assim, nas condições do presente estudo, a estimativa do potencial biótico desta espécie foi de 8.719.556 indivíduos.

O elevado valor do potencial biótico indica que em determinadas condições, como as de monocultura de eucalipto, especialmente em regiões mais quentes, ocasionalmente esta espécie possa apresentar aumentos populacionais acentuados, causando danos às florestas. Isso concorda com as ponderações de Zanuncio (1993) que considera esta espécie como uma praga secundária dessa cultura.
Do material examinado nas coleções foram localizados adultos coletados em todos os meses do ano no Rio Grande do Sul. Porem foram constatados com dois picos; um no início da primavera, principalmente em outubro e outro no verão, principalmente entre março e abril. Tais dados sugerem que neste Estado, em função das condições ambientais, esta espécie seja bivoltina como $A$. io, cujo período de desenvolvimento prolonga-se nos meses de inverno, provavelmente com a passagem por uma diapausa de inverno (Lemaire 2002) e possivelmente na fase de pupa como referido para A. coresus na Argentina (Bourquin 1944).

Três lagartas coletadas em Bento Gonçalves encontravamse cobertas por casulos típicos de braconídeos (Fig. 5) porém, antes de chegarem ao laboratório os microhimenópteros adultos já haviam emergido. Considerando a inexistência de informações sobre inimigos naturais desta espécie (Peigler 1994) este estudo indica a ocorrência de inimigos naturais que devem ser objeto de verificação mais precisa.

\section{AGRADECIMENTOS}

Aos curadores Eduardo J.E. Silva (MECB), Élvia E.S. Vianna (MUCP), Fernando R. Meyer (MAPA), Luciano Moura (MCNZ), Mirtes Melo (CAMB) e Vera R.S. Wollf (MRGC) pelo acesso e auxílio prestado durante o exame do material. À FAPERGS, pela concessão da Bolsa de Iniciação Científica (Proc. nº 02/508357) à segunda autora e pelo Auxílio Financeiro (Proc. $n^{\circ}$ 02/1739.6).

\section{REFERÊNCIAS BIBLIOGRÁFICAS}

BACKes, A.; M. NARdino. 2001. Nomes populares e científicos de plantas do Rio Grande do Sul. São Leopoldo, Unisinos, Coleções Fisionomia Gaúcha, $2^{\mathrm{a}}$ ed. 202p.

Biezanko, C.M.; A. Ruffinelli \& C.S. Carbonell. 1957. Lepidoptera Del Uruguay - Lista anotada de espécies. Revista de la Faculdad de Agronomía, Montevideo, 46: 1-152.

Biezanko, C.M.; A. Ruffinelli \& D. Link. 1974. Plantas y otras sustâncias alimentícias de las orugas de los lepidópteros uruguayos. Revista do Centro de Ciências Rurais, Santa Maria, 4 (2): 107-148.

Blest, A.D. \& T.S. Collett. 1965. Micro-eletrode studies of the medial protocerebrum of some Lepidoptera - I. Responses to simple, binocular visual stimulation. Journal of Insect Physiology, New York, 11 (8): 1079-1100.

Bourquin, F. 1944. IX - Observaciones sobre Automeris coresus (Boisduval), 1859 - Lep. Fam. Hemileucidae, p. 33-35. In: F. BouRQuin (ed.). Mariposas Argentinas - vida, desarrollo, costumbres y hechos curiosos de algunos lepidópteros argentinos. Buenos Aires, Ferrari Hermanos Bartolome Mitre, 223p.

Bourquin, F. 1948. Metamorfosis de Automeris naranja Schaus, 1898 (aurantiaca Weym.) (Lep. Het. Hemileucidae). Acta Zoologica Lilloana, Tucumán, 5: 69-80.

Butt, B.A. \& E. CAntu. 1962. Sex determination of 
lepidopterous pupae. Washington, USDA, 7p.

BuzZI, Z.J. 1994. Coletânea de nomes populares de insetos do Brasil. Curitiba, UFPR, 230p.

Collett, T.S. 1965. The control of the rocking response in hemileucine moths by sensory stimulation. Journal of Insect Physiology, New York, 11 (11): 1409-1425.

Corseuil, E.; A. Specht \& C. Lang. 2002. Saturniídeos (Lepidoptera, Saturniidae) registrados para o Rio Grande do Sul, Brasil. I. Hemileucinae. Biociências, Porto Alegre, 10 (2): 147-155.

Draudt, M. 1929. 12 - Familie Saturniidae, p. 713-827. In: A. SeITz (Ed.). Die Gross-Schmetterlinge der Erde. 6. Die Amerikanischen Spinner und Schwärmer. Stuttgart, Alfred Kernen, 1919-1944, 508p.

Dyar, H.G. 1890. The number of molts of lepidopterous larvae. Psyche, Cambridge, 5: 420-422.

Gardiner, B.O.C. 1982. A Silkmoth Rearer's Handbook. Hanworth, The Amateur Entomologists' Society, $3^{\text {rd }}, 255 \mathrm{p}$.

Guagliumi, P. 1957. Automeris illustris Wlk. (Lepid. Saturniidae), una nueva plaga de la caña de azucar en Venezuela. Agronomia Tropical, Maracay, 7 (3): 151-157.

Haddad, V. \& J.L.C. Cardoso. 2003. 22 - Erucismo e Lepidopterismo, p. 220-223. In: J.L.C. CARdoso; F.O.S. FranÇA; F.H.Wen; C.M.S. Málaque \& V. Haddad (Eds). Animais peçonhentos no Brasil - biologia, clínica e terapêutica dos acidentes. São Paulo, Sarvier, 468p.

JANZEN, D.H. 2003. How polyphagous are Costa Rican dry forest saturniid moth caterpillars?, p. 369-379. In: Y. BASSET; R. Kitching; S. Miller \& V. Novotny (Eds). Arthropods of Tropical Forests: Spatio-Temporal Dynamics and Resource Use in the Canopy. Cambrigde, Cambridge University Press, 490p.

KöHLER, P. 1935. Notas sobre Automerinae (Lep. Saturn.) argentinos. Revista de la Sociedad Entomologica Argentina, Buenos Aires, 7: 79-91.

Lemaire, C. 1996. Saturniidae, p. 28-49. In: J.B. Heppner (Ed.). Atlas of Neotropical Lepidoptera Checklist: Part 4B Drepanoidea - Bombycoidea - Sphingoidea. Gainesville, Association of Tropical Lepidoptera, L+87p.

Lemaire, C. 2002. The Saturniidae of America - Hemileucinae. Keltern, Goecke \& Evers, 3 vols, 1388p.

Lorini, L.M. 1999. A taturana: aspectos biológicos e morfológicos da Lonomia obliqua. Passo Fundo, EDIUPF, $67 \mathrm{p}$.

Lorini, L.M.; G.S. Rebelato \& J. Bonatti. 2004. Reproductive parameters of Lonomia obliqua Walker, 1855 (Lepidoptera: Saturniidae) in laboratory. Brazilian Archives of Biology and Technology, Curitiba, 47 (4): 575-577.

Mabilde, A.P. 1896. Guia práctico para os principiantes collecionadores de insectos, contendo a descrição fiel de perto de 1000 borboletas com 180 figuras lytographadas em tamanho, formas e desenhos conforme o natural. Estudo sobre a vida de insectos do Rio Grande do Sul e sobre a caça, classificação e conservação de uma coleção mais ou menos regular. Porto Alegre, Gunlach \& Schuldt, 238p.

Marinoni, R.C.; R.R.C. Dutra \& M.M. Casagrande. 1997. Levantamento da fauna entomológica no Estado do Paraná. III. Saturniidae (Lepidoptera). Revista Brasileira de Zoologia, Curitiba, 14 (2): 473-495.

Moraes, R.H.P. 2003. 21. Lepidópteros de importância médica, p. 211-219. In: J.L.C. Cardoso; F.O.S. França; F.H. Wen; C.M.S. Málaque \& V. Haddad (Eds). Animais peçonhentos no Brasil - biologia, clínica e terapêutica dos acidentes. São Paulo, Sarvier, 468p.

PARRA, J.R.P. \& M.L. HADDAD. 1989. Determinação do número de ínstares de insetos. Piracicaba, FEALQ, 49p.

Peigler, R.S. 1994. Catalog of parasitoids of Saturniidae of the World. Journal of Research on the Lepidoptera, Beverly Hills, 33: 1-121.

Santos, G.P.; J.C. Zanuncio \& T.V. Zanuncio. 1996a. Pragas do eucalipto. Informe Agropecuário, Belo Horizonte, 18 (185): 63-71.

Santos, G.P.; T.V. Zanuncio; O.S. Dias \& J.C. Zanuncio. 1996b. Biologia de Hylesia nanus (Walker) (Lepidoptera: Attacidae). Anais da Sociedade Entomológica do Brasil, Londrina, 25 (3): 479-482.

Silva, A.G.A.; C.R. Gonçalves; D.M. Galvão; A.J.L. Gonçalves; J. Gomes; M.M. SiLVA \& L. Simoni. 1968. Quarto Catálogo dos insetos que vivem nas plantas do Brasil: seus parasitos e predadores. Parte II, $1^{\circ}$ tomo, Insetos, hospedeiros e inimigos naturais. Rio de Janeiro, Ministério da Agricultura, $622 p$.

Silveira Neto, S.; O. Nakano; D. Barbin \& N.A. Villa Nova. 1976. Manual de ecologia dos insetos. São Paulo, Ceres, 419p.

Stone, S.E. 1991. Foodplants of World Saturniidae. Memoirs of the Lepidopteroists' Society, Los Angeles, 4: 1-186.

TABouret-Keller, A. 1958. Geotropism of saturnid larva Automeris illustris Walker. Comptes Rendus des Seances-Societe de Biologie et de Ses Filiales, Paris, 152 (5): 822-825.

YACK, J.E; M.L. SMith \& P.J. Weatherhead. 2001. Caterpillar talk: acoustically mediated territoriality in larval lepidoptera. Proceedings of the National Academy of Science - PNAS, Washington, 98 (20): 11371-11375.

ZanunCio, J.C. 1993. Manual de pragas em florestas Lepidoptera defoladores de eucalipto: biologia, ecologia e controle. Piracicaba, IPEF-SIF, 140p.

ZanunCiO, J.C.; M. Fagundes; M.S.S. AraúJo \& F.C. Evaristo. 1992. Monitoramento de lepidópteros, associados a plantios de eucalipto na Região de Açailândia (Maranhão), no período de agosto/90 a julho/91. Acta Amazônica, Manaus, 22 (4): 615-622.

ZanunCiO, J.C.; E.C. NASCIMENTO; J.F. GARCIA \& T.V. ZanUnCIO. 1994. Major lepidopterous defoliators of eucalypt in southeast Brazil. Forest Ecology and Management, Amsterdam, 65 (1): 53-63. 
Zanuncio, T.V.; J.C. Zanuncio; M.M.M. Miranda \& A.G.B. Medeiros. 1998a. Effect of plantation age on diversity and population fluctuation of Lepidoptera collected in Eucalyptus plantations in Brazil. Forest Ecology and Management, Amsterdam, 108 (1-2): 91-98.

Recebido em 17.VIII.2005; aceito em 16.V.2006.
Zanuncio, J.C.; J.A. Mezzomo; R.N.C. Guedes \& A.C. Oliveira. 1998b. Influence of strips of native vegetation on Lepidoptera associated with Eucalyptus cloeziana in Brazil. Forest Ecology and Management, Amsterdam, 108 (1-2): 85-90. 